\title{
Study of Field Misalignment in a Cavity Used for Atomic Clock Applications
}

\author{
Anton E. Ivanov ${ }^{1}$, Christoph Affolderbach ${ }^{2}$, Gaetano Mileti ${ }^{2}$ and Anja K. Skrivervik ${ }^{1}$ \\ ${ }^{1}$ Laboratoire d'Électromagnétisme et d'Acoustique (LEMA), École Polytechnique Fédérale de Lausanne (EPFL), \\ Lausanne, Switzerland, E-mail: antonevgeniev.ivanov@epfl.ch \\ ${ }^{2}$ Laboratoire Temps-Fréquence, University of Neuchâtel, Neuchâtel, Switzerland
}

\begin{abstract}
In vapor cell atomic clocks the atom-field interaction is typically obtained inside a microwave cavity resonator in which the microwave driving field together with a static magnetic field and an optical field are applied to excite the atoms. These fields are generally well-controlled, mutually aligned to a common quantization axis. Since the exploited atomic transition is sensitive to any potential axis misalignment, the performance of the clock can also be affected. We study the effect of such misalignment for the case of a cylindrical cavity used in vapor-cell atomic clocks, taking into account the misalignments of the optical detection field and the static magnetic field required for the atomic transition. Both the geometry of the cavity and the factors contributing to losses can play role in the degradation of the signal and are taken into account in the misalignment problem discussed.
\end{abstract}

\section{INTRODUCTION}

Double resonance (DR) vapor cell atomic clocks are an important class of compact atomic frequency standards, mainly applied in telecommunications and space applications [1]. Both in view of performance and size they can be considered situated between the large atomic fountains and the miniature mass-produced quartz oscillators. In such a DR cell clock, an atomic transition in the microwave region is employed as an inherently stable frequency reference for stabilizing the frequency of a quartz oscillator. It is driven by the standing-wave magnetic field provided by a microwave cavity tuned to a chosen transition (ex. $6.835 \mathrm{GHz}$ for the usually employed ${ }^{87} \mathrm{Rb}$ ). To selectively detect the atomic reference transition, a static magnetic field (C-field) and an additional optical field need to be applied to the atoms inside the cavity. For a stable clock signal it is of crucial importance that both these fields are well-controlled and perfectly aligned to the direction of the microwave magnetic field (the $z$ coordinate for the typically used cylindrical $\mathrm{TE}_{011}$ cavity figure 1a).

Recently very high stability has been achieved for vapor clocks - reaching fractional frequency instability of few $10^{-15}$ (in terms of Allan deviation), over time scales (integration time) $\tau \geq 10^{4} \mathrm{~s}$ [2]. For the implementation of such highperformance clocks, the microwave cavity is recognized as a crucial component and its quality has a high impact on the clock performance limitations [3]. In the evolution of the atomic clocks based on cavity resonators the design of the cavity has been the focus of research mainly connected to improving the compactness [4], the homogeneity of the magnetic field [5], but also characterizing the thermal and mechanical stability and the effect of losses [3, 6]. While a combination of analytical, lumped-method approach and fullwave simulations is sufficient to model even complex cavities used in such clocks, as for instance the loop-gap geometry [4], the experimental verification of the cavity performance has been limited to measurements traditionally based on the Zeeman spectra corresponding to the atomic transitions or the Rabi oscillation method when estimation of the field homogeneity is required [3]. Recently a new characterization technique allowed obtaining spatially-resolved information for the microwave field inside the cavity [7, 8]. The method is non-perturbative and uses the light and microwave interaction with the atoms to deduce the field distribution in the active volume of the cavity. It results in $2 \mathrm{D}$ images averaged along the direction of the light, proportional to the strength of the microwave magnetic field driving the atomic resonance transitions - figure $1 \mathrm{~b}$. Such images are very informative because they reveal field inhomogeneities that can arise from manufacturing imperfections or misalignment between the fields required for the operation of the clock. These effects have the potential to degrade the performance of the clock and thus require further attention.

In our study, aiming to support interpretation of such an experiment, we theoretically investigate the circular $\mathrm{TE}_{011}$ cavity that is traditionally used in atomic vapor cell clocks working in the double resonance (DR) regime. The required fields (microwave field, static magnetic field and optical beam) are considered misaligned and the corresponding 2D field profiles that are physically comparable to a field imaging experiment are calculated. The effect of losses arising from non-PEC boundary conditions is investigated and linked to the corresponding field quantities driving the atomic transitions.

\section{VAPOR CELL FREQUENCY STANDARDS}

\section{A. Clock Operation in the DR regime}

In vapor cell clocks based on DR a ground state transition in the hyperfine structure of ${ }^{87} \mathrm{Rb}$ is used as a stable frequency reference (e.g. the $\left|F=1, m_{F}=0\right\rangle \leftrightarrow\left|F=2, m_{F}=0\right\rangle$ in the $5 S_{1 / 2}$ ground state of ${ }^{87} \mathrm{Rb}$ ), referred to as "clock transition". The $\mathrm{Rb}$ atoms are in thermal vapor phase, and are contained in a dielectric cell enclosed in the center of a microwave cavity. Inside, the atoms interact with three well-controlled EM fields: a homogeneous static magnetic field (C-field) required for separating the clock transition from other transitions involving hyperfine levels with $\mathrm{m}_{\mathrm{F}} \neq 0$, via Zeeman splitting, a pumping light field (for example - resonant to the Rb D2-line, $v_{1}=384.23 \mathrm{THz}$ ) excites the atoms and creates population 
inversion, and a microwave magnetic field provided by the cavity and tuned to the required clock transition $\left(\nu_{\mathrm{Rb}} \approx 6.835\right.$ $\mathrm{GHz}$ ). The useful signal of the clock is obtained by a photodetector, using the absorption of light at the condition of double resonance $-\left(v_{1}\right.$ and $\left.v_{\mathrm{Rb}}\right)$. In Figure $1 \mathrm{a}$ we show a simplified scheme of such a clock.
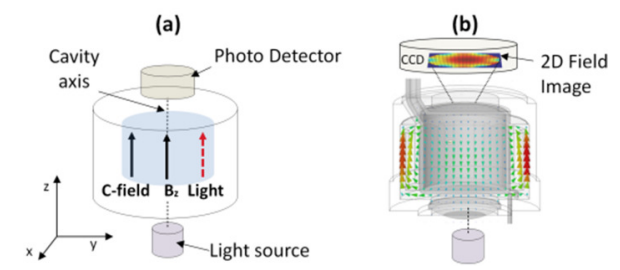

Fig. 1 (a) The cavity is shown with the dielectric cell (blue). Openings at the top and bottom allow for the light to penetrate the vapor. Ideally inside the active zone of the cavity the C-field and the light beam are perfectly aligned along the direction of the axis thus to the $\mathrm{H}_{\mathrm{z}}$ component for the considered $\mathrm{TE}_{011}$ mode. (b) Field imaging experiment.

\section{B. Atomic transitions driven by the cavity}

In the above-explained DR regime, the atomic response can be characterized by three transitions $\sigma_{-}, \sigma_{+}$and $\pi$ all driven for the chosen $\mathrm{TE}_{011}$ mode (figure 2).

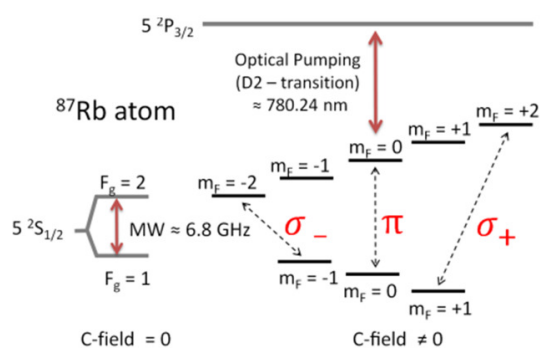

Fig. 2 Energy levels of ${ }^{87} \mathrm{Rb}$ ground state and $5^{2} \mathrm{P}_{3 / 2}$ excited state (D2 line). The C-field breaks the initially degenerate levels (shown on the left) by defining a quantization axis - chosen along the $\mathrm{z}$ direction of the cavity (figure 1). The Zeeman sub-levels are shown on the right. Possible transitions driven by the cavity link the quantum states $\left|F_{\mathrm{g}}=1, \mathrm{~m}_{\mathrm{F}}=-1\right\rangle \rightarrow|2,-2\rangle,|1,0\rangle \rightarrow|2,0\rangle$, and $|1,+1\rangle \rightarrow|2,+2\rangle$ and due to the change of $m_{F}$ quantum number are classified as $\sigma_{-}, \pi, \sigma_{+}$ transitions, respectively.

Classically they are seen as magnetic dipole-flip transitions oscillating with Rabi frequencies and following the formalism [9] they can be written as:

$$
\begin{aligned}
& \Omega_{-}=-\sqrt{3} \frac{\mu_{B}}{\hbar} B_{-} e^{j \phi_{-}}, \\
& \Omega_{\pi}=-\frac{\mu_{B}}{\hbar} B_{\pi} e^{j \phi_{\pi}}, \\
& \Omega_{+}=\sqrt{3} \frac{\mu_{B}}{\hbar} B_{+} e^{j \phi_{+}},
\end{aligned}
$$

where $\mu_{\mathrm{B}}$ is the Bohr magneton, $\hbar$ is the reduced Planck constant; $B_{-}, B_{\pi}, B_{+}$are the scalar projections of the microwave field in the complex circular basis $\hat{b}_{-}, \hat{b}_{\pi}, \hat{b}_{+}$:

$$
\begin{aligned}
& B_{-} e^{j \phi_{-}}=\hat{b}_{-} \cdot \vec{B}_{x y z}=\frac{1}{\sqrt{2}}\left(B_{x}+j B_{y}\right), \\
& B_{\pi} e^{j \phi_{\pi}}=\hat{b}_{\pi} \cdot \vec{B}_{x y z}=B_{z}, \\
& B_{+} e^{j \phi_{+}}=\hat{b}_{+} \cdot \vec{B}_{x y z}=\frac{1}{\sqrt{2}}\left(B_{x}-j B_{y}\right) .
\end{aligned}
$$

Here $\vec{B}_{x y z}$ is the magnetic (induction) field of the lossless cavity in the Cartesian basis aligned to the cavity axis. The corresponding cavity components $B_{x}, B_{y}, B_{z}$ can be analytically obtained in a standard way for the steady-state solution where in the general case each is characterized by its own phase: $\left|B_{i}\right| e^{j \phi i}=R_{i}+j I_{i}, i$ element of $x, y, z$. Physically, the quantities on the left side correspond to the amplitude and phase of the magnetic fields driving the resonance transitions - referred to as "driving" fields. The useful clock signal is obtained from the $\pi$ - transition driven by the magnetic field component aligned to the direction of the C-field (by $B_{z}$ in the case of perfectly aligned cavity), while the two additional transitions $\sigma_{-}$and $\sigma_{+}$are driven by the left and right circularly polarized driving fields $\left(B_{-}\right.$and $B_{+}$) and can thus be used to assess experimentally these field components. Neglecting the effect of the feed and the openings, there is no phase difference between the components of the magnetic field inside the cavity and for the magnitude we obtain:

$$
\left|B_{-}\right|=\left|B_{+}\right|=\frac{1}{\sqrt{2}} \sqrt{R_{x}^{2}+I_{x}^{2}+R_{y}^{2}+I_{y}^{2}} .
$$

\section{Figure of merit}

One way to improve the stability of the clock is to increase the signal-to-noise ratio of the useful signal by increasing the total number of detected atoms that are resonant only to the selected clock transition. In figure 3 we show an example of the resulting Zeeman spectrum including all possible transitions for the case of the well-aligned cavity [5]. In order to estimate the fraction of microwave power in the cavity that is effectively coupled to the beneficial clock transition, the Field Orientation Factor (FOF) can be used [5]:

$$
\xi=\frac{\int B_{z}^{2} d v}{\int\left\|\vec{B}_{x y z}\right\|^{2} d v}=\frac{\int B_{\pi}{ }^{2} d v}{\int\left\|\vec{B}_{\sigma_{-} \sigma_{+} \pi}\right\|^{2} d v}
$$

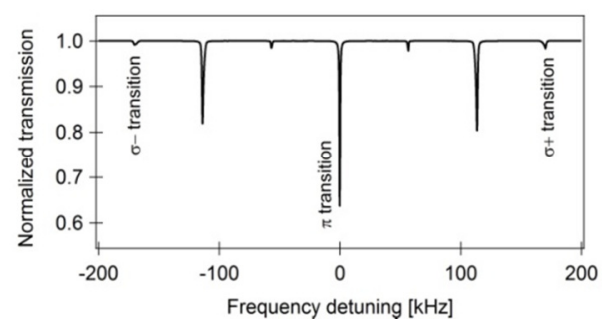

Fig. 3 Zeeman transitions, the central line corresponds to the required clock transition for which the cavity needs to be designed to maximize.

It can be obtained from the experimental spectra and it is a figure of merit that can be used to characterize the performance of the cavity.

\section{Description of the general misalignment problem}

In a real clock implementation, the EM fields interacting with the atoms can be misaligned with respect to each-other and have the potential to degrade the performance of the clock by increasing the coupling to the unwanted $\sigma_{-}$and $\sigma_{+}$ transitions. We consider a perfectly collimated (parallel) light beam of uniform intensity profile, emitted by a laser situated at the bottom opening of the cavity. The walls of the cavity are non-reflecting for the light and the detector is fixed and 
perfectly aligned to the cavity axis. The C-field is considered to be fully-homogenous everywhere inside the cavity - the fringing field is neglected and therefore the quantization axis defined by the $\mathrm{C}$-field vector is equal for all atoms. The effects of the $\mathrm{Rb}$ vapor and the dielectric cell are neglected. The microwave magnetic field is calculated with respect to a coordinate system aligned to the central axis of the cavity, it is decomposed along the tilted $\mathrm{C}$-field direction and the driving fields $B_{-}, B_{+}$and $B_{\pi}$ are obtained (figure 4 ).
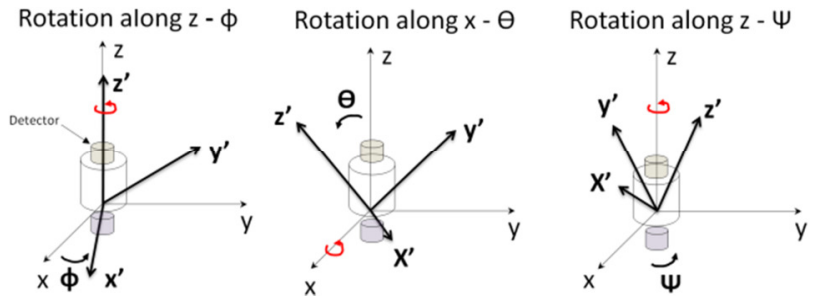

Fig. 4 The cavity is fixed to a coordinate system xyz. The coordinate system $x^{\prime} y^{\prime} z^{\prime}$ is obtained by three consecutive rotations related to the angles $\phi, \theta, \psi$ each corresponding to rotation along a particular axis $(z-x-z$ rotation convention, extrinsic rotation). The quantization axis is considered to be along $z^{\prime}$. The direction of the angles shown corresponds to the negative values.

The amplitude of the signal detected at a given detector pixel is influenced by the optical density due to the atomic absorption (proportional to the amplitude of the corresponding driving field), averaged along the direction of the light beam [8]. A misalignment will result in a horizontal displacement of the averaging direction, spread of the shape of the peak of the detected field and reduced amount of the useful signal. To model this effect we apply a 3D shearing, performed with respect to the $z$ axis (figure 5). The effect of this transformation is to displace the $x$ and $y$ coordinates by an amount that is proportional to the $z$ coordinate, leaving $z$ unchanged.

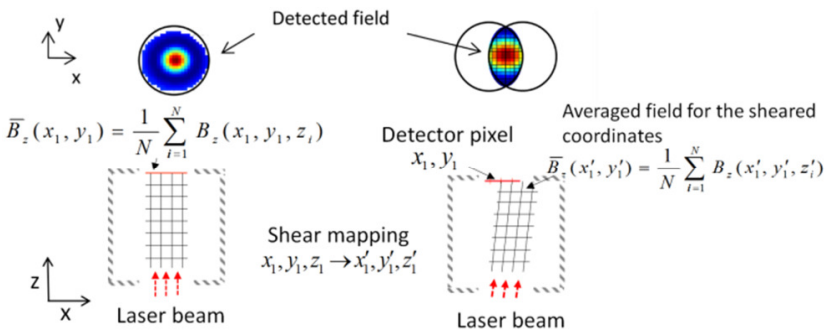

Fig. 5 The displaced direction of the laser light is obtained by applying shear mapping to the initial rectangular grid used; $x^{\prime}, y^{\prime}, z^{\prime}$ are the coordinates of the sheared $3 \mathrm{D}$ grid used to obtain the fields.

\section{III.RESULTS AND DISCUSSION}

The results shown in this part are obtained for a cavity with dimensions: radius $-R=0.03 \mathrm{~m}$, height $-L=0.0485 \mathrm{~m}$, resonance frequency $f=6.834 \mathrm{GHz}, \mathrm{TE}_{011}$ mode. The 2D, averaged field profiles correspond to the radius of the laser beam $-0.015 \mathrm{~m}$ or the radius of the cavity.

\section{A. Driving fields in the perfectly aligned cavity}

In this case, the maximum for $B_{\pi}$ is found in the center with fields driving the $\sigma$ transitions having minimum along the central axis - favorable since the main part of the clock signal is obtained from the atoms in the central region. In figure 6 we show the driving fields in the cavity and in 7 the obtained field profiles.
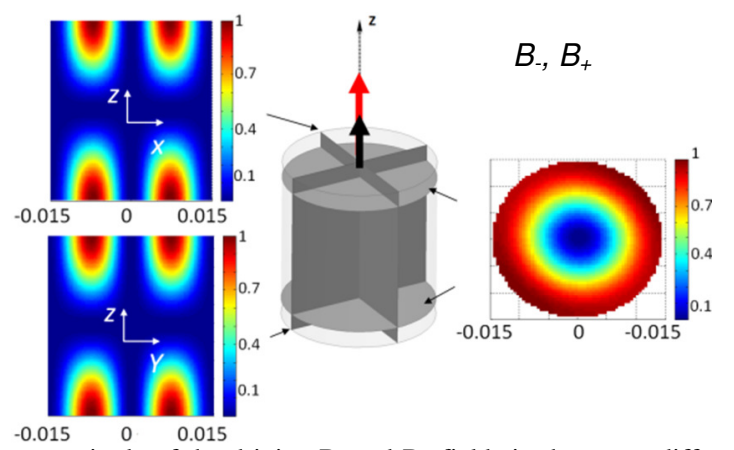

Fig. 6 The magnitude of the driving $B_{\text {. }}$ and $B_{+}$fields is shown on different planes for the aligned cavity, normalized to the maximum. The directions of the laser beam and the $\mathrm{C}$-field are indicated by the red and black arrows at the top of the cavity.

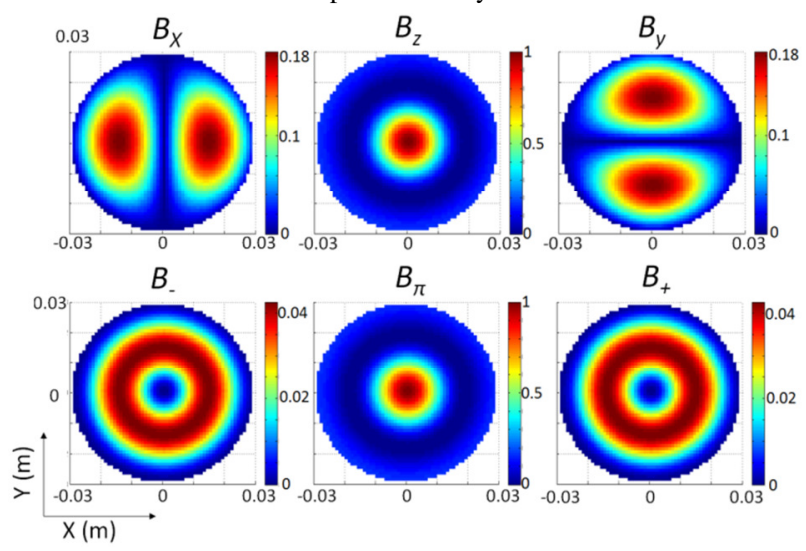

Fig. 7 2D averaged field profiles corresponding to the components of the magnetic field obtained for the cavity (upper row) and to the related driving fields that interact with the atoms (lower row). The amplitudes are normalized to the maximum (found in the center for $\mathrm{TE}_{011}$ ), and each component is color-coded separately.

\section{B. Misalignment of the $C$-field}

When $\theta=0$, a rotation at $\phi$ and $\psi$ is identical, it will affect only the $B_{x}$ and $B_{y}$ components $-B_{z}$ is azimuthally symmetric, since the driving $B_{-}$fields and $B_{+}$are invariant to rotation $\theta=0, \phi \neq 0, \psi \neq 0$. The symmetry is broken in the rotation $\theta \neq 0$ which is easily seen for the driving $B_{-}$and $B_{+}$fields shown inside the cavity in figure 8 and for the averaged profiles in figure 9. As a result of the rotation, the field at the top and the bottom of the cavity will be somewhat mirrored (fig 8 - right). However the effect will be averaged along the direction of the light beam, resulting in the slightly asymmetric field images (figure 9). Once the azimuthal symmetry is broken the integrated images can be further rotated by $\psi \neq 0$, but are invariant to rotation $\phi \neq 0$. The images obtained for $B_{-}$and $B_{+}$are always identical regardless the direction of the $\mathrm{C}$-field chosen. The amount of clock signal degradation, due to increased coupling of $B_{-}$and $B_{+}$is determined by the $\theta$ angle only and thus FOF (figure 10) is invariant to rotation at $\phi$ and $\psi$. Any misalignment of the Cfield will equally increase the coupling to both the $\sigma_{-}$and $\sigma_{+}$ transitions. 


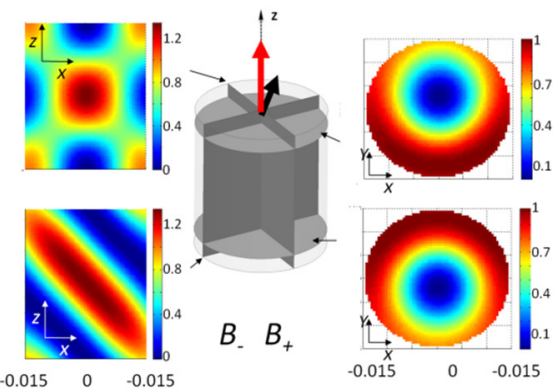

Fig. 8 The magnitude of the driving $\mathrm{B}_{\text {. and }} \mathrm{B}_{+}$fields is shown for two different longitudinal planes (left) and two transverse planes (right). The directions of the laser beam and the C-field are indicated by the red and black arrows at the top of the cavity. The fields are normalized to the maximum amplitudes found in the case of perfectly aligned cavity.

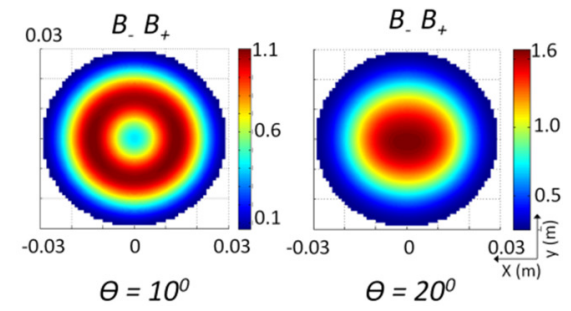

Fig. 9 2D averaged field profiles, calculated for the misaligned C-field, normalized to the maximum amplitudes found in the case of the perfectly aligned cavity. Each component is color-coded separately.

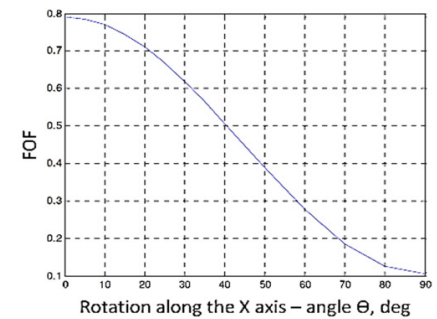

Fig. 10 Variation of the FOF as a function of the $\theta$ rotation.

\section{Rotation of the light field}

For the generalized misalignment we report the results in the $2 \mathrm{D}$ diagram shown in figure 11 . The perfectly aligned cavity is thus situated in the center of the diagram.

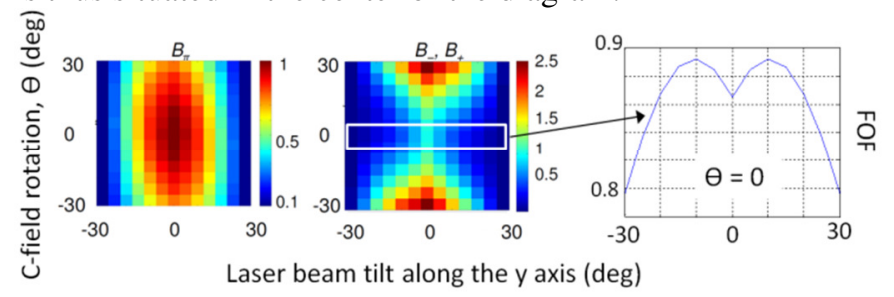

Fig. 11 Total amount of the driving fields found inside the volume of the cavity: - $\mathrm{B}_{\pi}, \mathrm{B}_{-} \mathrm{B}$; normalized to the total amount found for the case of perfectly-aligned cavity. FOF (right) is shown for a sweep of laser light angles along the y direction and for $\mathrm{C}$-field at $\theta=0$.

The calculations show that although the misalignment will in any case decrease the useful signal, small misalignment of the laser direction leads to a slight increase of the FOF. This is an interesting geometrical effect attributed to the fact that along the radius $B_{-}$and $B_{+}$have opposite slope to $B_{\pi}$.

\section{Influence of the perturbation field}

We have used a full-wave simulation performed in HFSS in order to deduce the approximated effect that impedance boundary conditions have on the driving fields (figure 12).

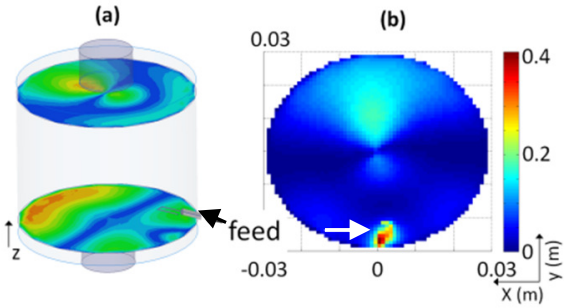

Fig. 12 (a) Cavity modelled with feed and openings. (b) The averaged magnitude of $\mathrm{B}_{-}$and $\mathrm{B}_{+}$is obtained from the perturbation fields arising from the impedance boundary condition and is normalized to the corresponding fields found in the center of the lossless cavity.

We note that this is a secondary effect (the total contribution is less than 1\%) that will be washed-out by the main (unperturbed) field. However, from the simulations it is interesting to see that there is an increase of the $B_{-}$and $B_{+}$ close to the center of the cavity.

\section{CONCLUSION}

In this study we theoretically characterize the impact of potential C-field and light field misalignment in the implementation of a vapor cell atomic clock based on $\mathrm{TE}_{011}$ cavity. The effect of arbitrary misalignment is quantified via the FOF that is in principle experimentally accessible. The various cases are illustrated using $2 \mathrm{D}$ field profiles averaged along the direction of the pumping light. FEM is used to obtain the perturbation field solution to non-PEC boundary conditions and link them to the fields driving the atomic resonance. Our study is relevant in line with the newly developed non-perturbative field characterization techniques.

\section{REFERENCES}

[1] J. Camparo, Phys. Today 60, 33, 2007

[2] S Micalizio, C E Calosso, A Godone and F Levi, "Metrological characterization of the pulsed $\mathrm{Rb}$ clock with optical detection", Metrologia 49 (2012) 425-436

[3] A. Godone, S. Micalizio, F. Levi, and C. Calosso, "Microwave cavities for vapor cell frequency standards", Rev. Sci. Instrum., vol. 82, no. 7, 074703, July 2011.

[4] C. Stefanucci, T. Bandi, F. Merli, M. Pellaton, C. Affolderbach, G. Mileti, and A. K. Skrivervik, "Compact microwave cavity for high performance rubidium frequency standards". Rev. Sci. Instrum., vol. 83, 104706, Oct. 2012.

[5] G. Aviv, "Two-photon coherent manipulation of an atomic two-state system," Ben-Gurion University of the Negev, 2009.

[6] R. Li and K. Gibble, "Phase variations in microwave cavities for atomic clocks, "Metrologia 41, 376-386 (2004).

[7] A. Horsley, G.-X. Du, M. Pellaton, C. Affolderbach, G. Mileti, and P. Treutlein, "Imaging of Relaxation Times and Microwave Field Strength in a Microfabricated Vapor Cell", Phys. Rev. A 88, 063407, Dec. 2013.

[8] A. E. Ivanov, T. Bandi, Guan-Xiang Du, A. Horsley, C. Affolderbach, P. Treutlein, G. Mileti, and A. K. Skrivervik. "Experimental and Numerical Studies of the Microwave Field Distribution in a Compact Magnetron"-28th European Frequency and Time Forum (EFTF), Neuchatel, Switzerland, June 22-26, 2014.

[9] P. Böhi, M. F. Riedel, T. W. Hänsch, and P. Treutlein, "Imaging of microwave fields using ultracold atoms", Appl. Phys. Lett., vol. 97, no.5, 051101, Aug. 2010. 